Historic, Archive Document

Do not assume content reflects current scientific knowledge, policies, or practices. 



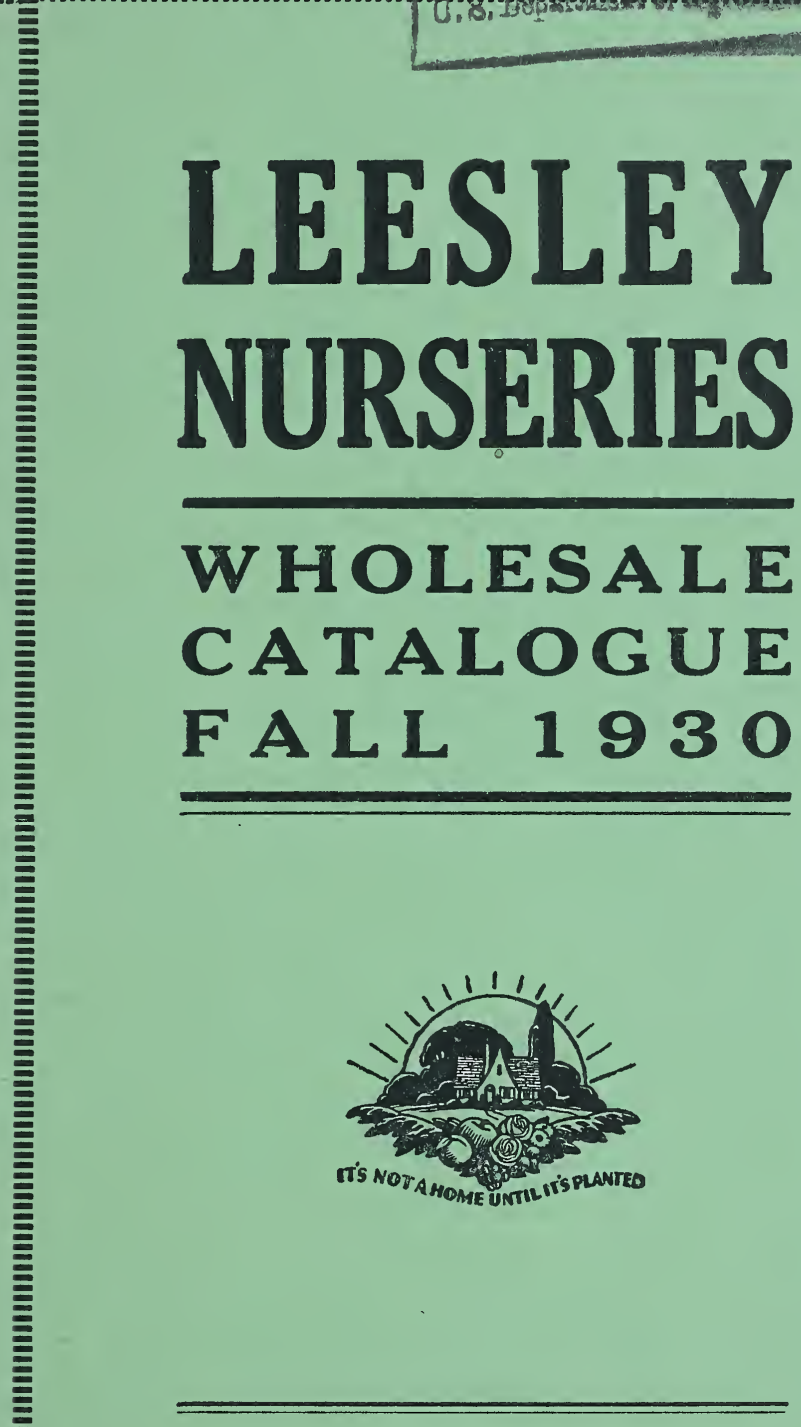

\section{LIBERTYVILLE, ILLINOIS}

Tel. Libertyville 172 



\section{WHOLESALE CATALOGUE}

$$
\overline{\text { FA L L } 1930}
$$

\section{E E S L E Y NURSERIES}

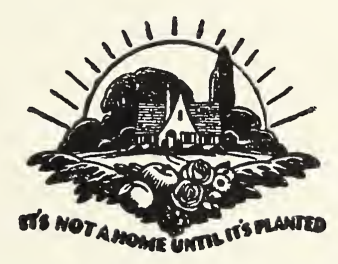

LIBERTYVILLE, ILLINOIS

Tel. Libertyville 172 


\section{E E S L E Y N U R S E R I E S}

Our Nurseries are located two miles northwest of the town on Milwaukee Ave. or Route 21 , which is now paved to our entrance.

\section{RE M I T T A N C E S}

should be made by Postal or Express Money Orders, Bank Drafts or by Registered Letters, made payable to the order of LEESLEY NURSERIES.

\section{$\begin{array}{llllll}P & R & I & C & E & S\end{array}$}

do not include packing, which will be charged for at cost. Five will be furnished at the 10 rate and 25 at the 100 rate. No charge for delivery to the express companies or railroad. Certificate of inspection furnished with each shipment. 


\section{ORNAMENTAL TREES}

ACER dasycarpum (Silver-leaved Maple)

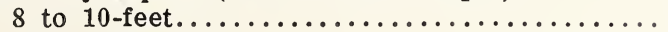

$11 / 4$ to $11 / 2$-inch $\ldots \ldots \ldots \ldots \ldots \ldots \ldots \ldots \ldots \ldots$

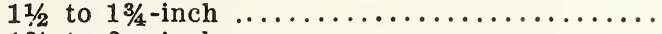

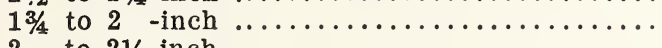

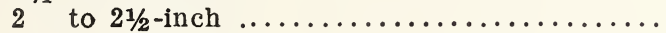

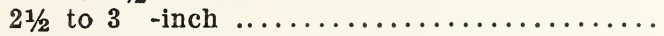

3 to $31 / 2$-inch $\ldots \ldots \ldots \ldots \ldots \ldots \ldots \ldots \ldots \ldots$

$31 / 2$ to 4 -inch $\ldots \ldots \ldots \ldots \ldots \ldots \ldots \ldots \ldots$

dasy. Wieri (Wier Cutleaf Maple)

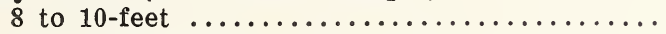

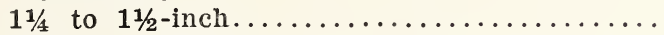

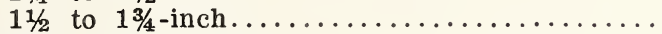

Each

Per 10

Per 100

$\$ 1.00 \$ 8.50$

$\$ 75.00$

$1.50 \quad 12.50$

$2.00 \quad 17.50$

$2.25 \quad 20.00$

$2.75 \quad 25.00$

$3.00 \quad 27.50$

4.50

6.50

$1.50 \quad 12.50$

$1.75 \quad 15.00$

$2.00 \quad 17.50$

Ginnala (Amur Maple)

3 to 4 -feet........................... $\quad .75$

\subsection{0}

platanoides (Norway Maple)

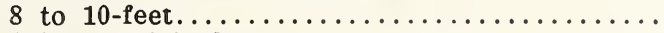

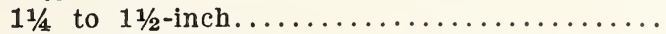

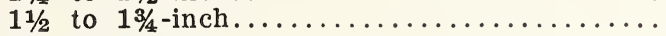

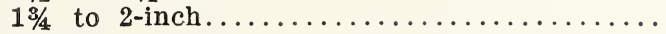

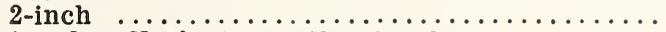

plat. schwedleri (Schwedler Maple)

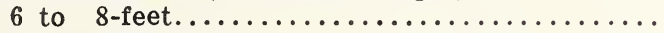

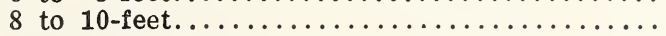

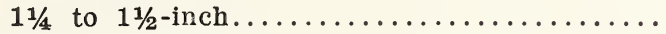

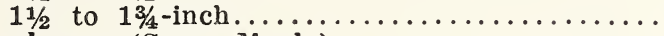
saccharum (Sugar Maple)

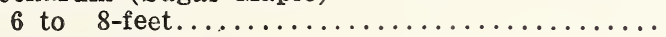

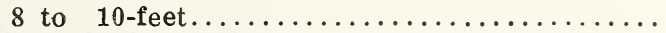

AESCULUS hippocastanum (Horse Chestnut)

2 -inch

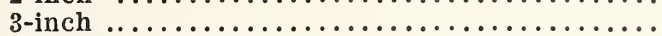

20.00

3.00

27.50

35.00

$4.50 \quad 40.00$

$5.50 \quad 50.00$

100.00

150.00

175.00

225.00

$2.25 \quad 20.00$

$3.00 \quad 27.50$

$4.00 \quad 37.50$

$4.75 \quad 45.00$

$1.50 \quad 12.50$

$2.00 \quad 17.50$

7.00

12.00

AILANTHUS glandulosa (Tree of Heaven)

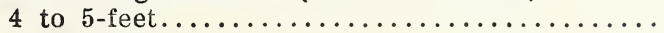

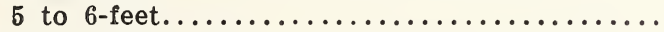

6 to 8 -feet.

$.60 \quad 5.00$

6.00

45.00

.75

.85

7.50

175.00

250.00

325.00

375.00

BRTULA alba laciniata (Cutleaf Weep. Birch)

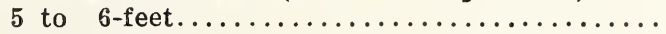

$1.50 \quad 12.50$

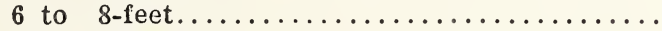

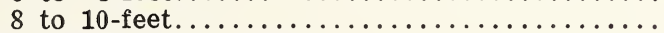
papyrifera (Canoe Birch)

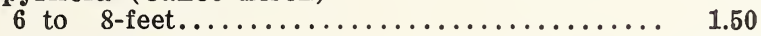

8 to 10 -feet......................... 2.00

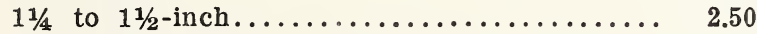

2 to $21 / 2$-inch..................... 3.50

CATALPA bungei (Umbrella Tree)

1 -year -5 to 6 -foot stems ............... 1.25

10.00

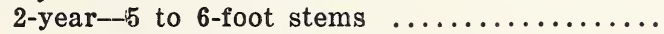

1.50

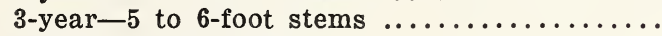

1.75

4-year-6-foot stems ................. 2.50

12.50

15.00

speciosa (Western Catalpa)

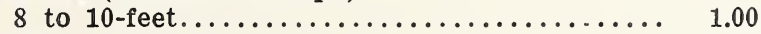

22.50

55.00

$11 / 4$ to $11 / 2-$ inch

7.50

1.25

$11 / 2$ to 2 -inch...................... 1.75

10.00

2 to $21 / 2$-inch...................... 2.25

15.00

20.00

CERCIS Canadensis, Tree form (American Red Bud)

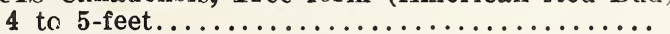

FRAXINUS americana (American White Ash)

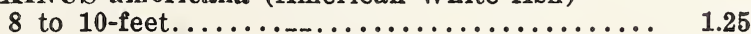

10.00

$11 / 4$ to $11 / 2$-inch. 
$11 / 2$ Each

$11 / 2$ to $13 / 4$-inch...................... $\$ 2.00$

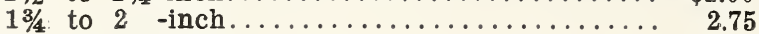

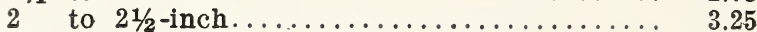

GLEDITSIA triacanthos (Honey Locust)

\begin{tabular}{|c|c|c|}
\hline$\ldots \ldots \ldots$ & .80 & 7.50 \\
\hline to 10 -feet............ & 1.25 & 10.00 \\
\hline $11 / 4$ to $11 / 2-i n c h \ldots \ldots \ldots \ldots \ldots \ldots \ldots \ldots$ & 1.50 & 12.50 \\
\hline 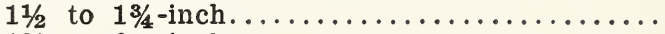 & 1.75 & 15.00 \\
\hline- inch $\ldots \ldots \ldots \ldots \ldots \ldots \ldots \ldots \ldots$ & 2.00 & 17.50 \\
\hline 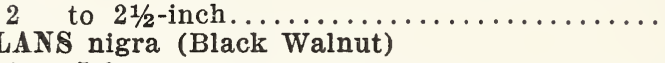 & 2.75 & 25.00 \\
\hline dioica (Canadonsis) & .75 & 6.0 \\
\hline .... & .6 & \\
\hline
\end{tabular}

MAGNOLIA soulangeana

2 to 3 -feet......................

POPULUS alba bolleana

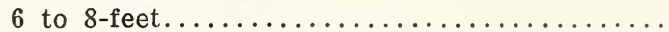

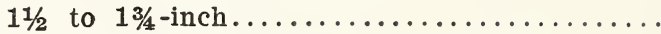

alba (Silver leaved Poplar)

8 to 10 -feet....................... 1.25

eugenie monolifera (Carolina Poplar)

$11 / 4$ to $11 / 2$-inch......................

$11 / 2$ to $13 / 4-i n c h \ldots \ldots \ldots \ldots \ldots \ldots \ldots \ldots \ldots$

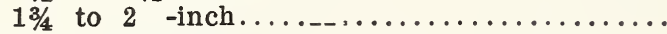

2 to $21 / 2-i n c h . \ldots \ldots \ldots \ldots \ldots \ldots \ldots \ldots$

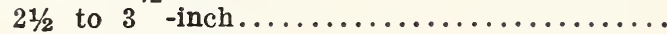

3 to $3 \frac{1 / 2}{}-i n c h \ldots \ldots \ldots \ldots \ldots \ldots \ldots \ldots$

nigra italica (Lombardy Poplar)

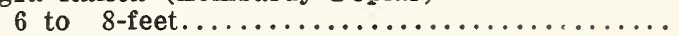

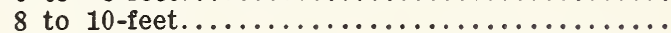

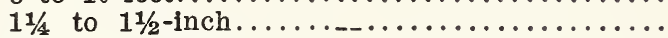

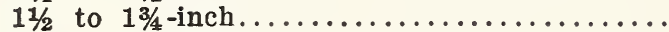

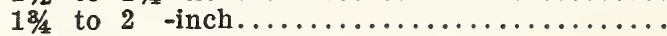

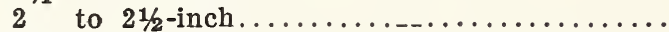

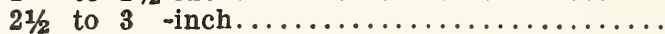

simoni (Chinese Poplar)

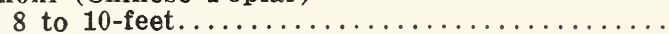

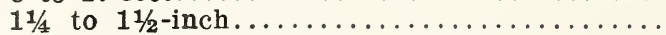

PTELIA trifoliata (Hoptree)

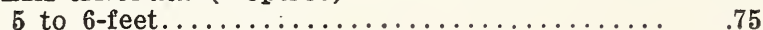

QUERCUS palustris (Pin Oak)

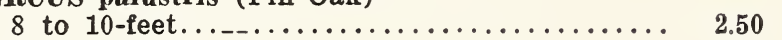

rubra (Red Oak)

6 to 8 -feet......................... 2.00

ROBINIA pseud acacia (Black Locust)

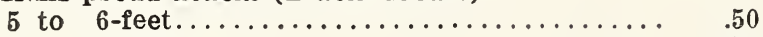

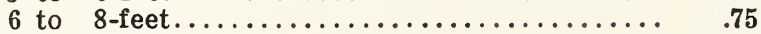

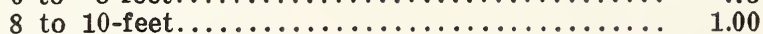

hispida (Rose-acacia)

2 to 3 -feet....................... 50

SALIX vitellina britzensis (Red Willow)

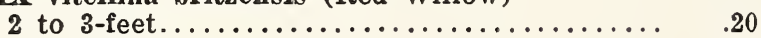

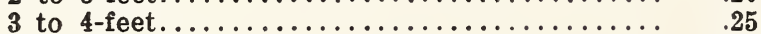

4 to 5 -feet $\ldots \ldots \ldots \ldots \ldots \ldots \ldots \ldots \ldots \ldots \ldots \ldots \ldots \ldots . \ldots \ldots \ldots$

caprea (Pussy Willow)

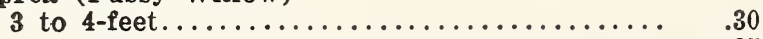

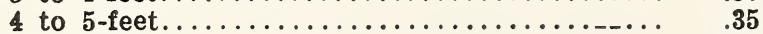

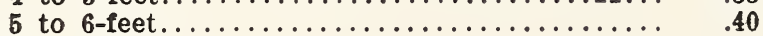

niobe (Weeping)

5 to 6 -feet......................... 50

pentandra laurifolia (Laurel Willow)

4 to 5 -reet
Per 10

$\$ 17.50$

25.00

30.00

7.50

10.00

15.00

17.50

6.00

5.00

\section{.50}

20.00

11.00

90.00

4.50

40.00

$8.50 \quad 75.00$

$11.00 \quad 100.00$

$16.00 \quad 150.00$

22.50

30.00

$4.00 \quad 35.00$

$6.00 \quad 50.00$

$8.50 \quad 75.00$

$11.00 \quad 100.00$

$16.00 \quad 150.00$

20.00

25.00

6.00

7.50

6.00

5

4.00

6.00

7.50

4.00

$\begin{array}{ll}1.75 & 15.00 \\ 2.00 & 17.50 \\ 3.00 & 25.00\end{array}$

$2.50 \quad 20.00$

$3.00 \quad 25.00$

$3.50 \quad 30.00$

4.00

.40

3.50

30.00 
vitellina (Golden Willow)

3 to 4 -feet. . . . ................... $\$ 0.30$

4 to 5 -feet...............................

5 to 6 -feet.................................

viminalis (Basket Willow)

4 to 5 -feet......................... .30

SORBUS aucuparia (European Mountain Ash)

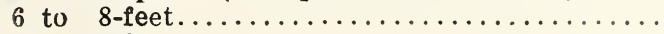

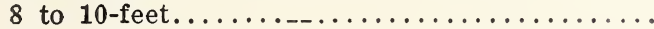

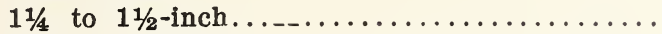

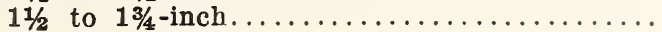

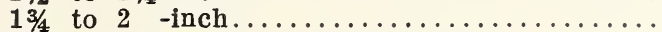

Americana (American Mountain Ash)

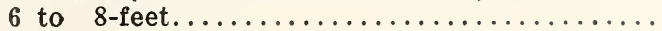

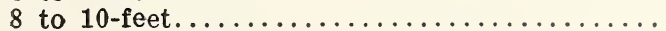

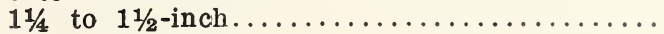

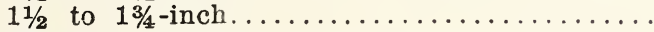

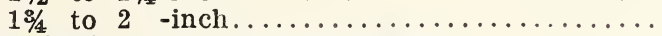

quercifolia (Oakleaf Mountain Ash)

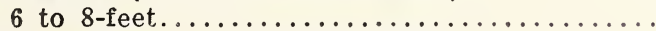

TILIA americana (American Linden)

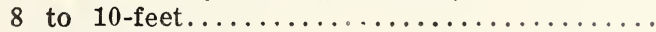

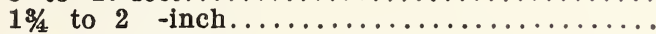

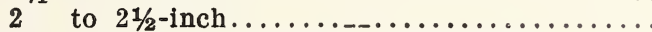

ULMUS americana (American White Elm)

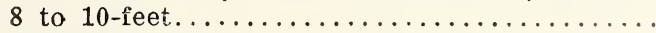

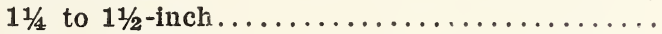

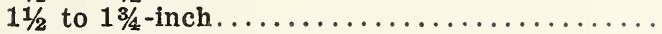

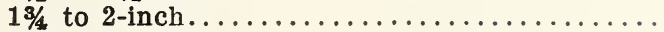

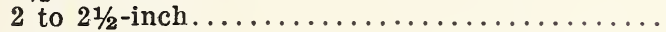

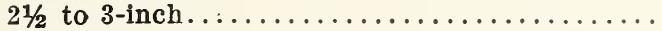

3 to $31 / 2$-inch

$31 / 2$ to 4 -inch

5 -inch

Moline (Moline Elm)

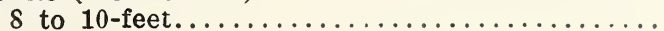

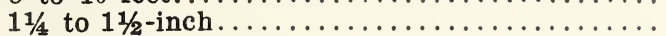

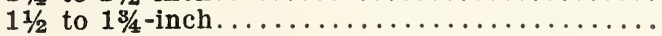

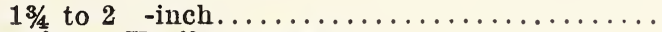

Americana Urnii (Vase Elm)

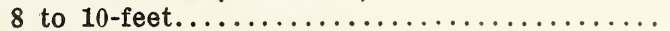

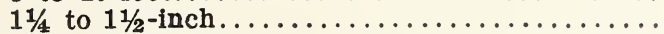

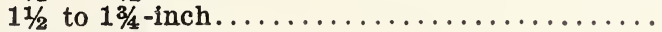

6 to 8 -feet..........

1.25

1.75

3.00

3.50

9.00

14.00

16.00

1.50

2.00

2.25

2.75

1.75

2.25

2.75

1.25

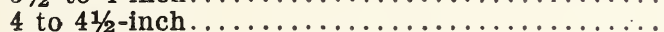

pumila (Chinese Elm)

Per 10

Per 100

$\$ 2.50$

3.00

3.50

2.50

$\begin{array}{rr}1.00 & 7.50 \\ 1.25 & 10.00\end{array}$

$1.75 \quad 15.00$

$2.25 \quad 20.00$

$2.75 \quad 25.00$

$1.50 \quad 12.50$

$1.75 \quad 15.00$

$2.25 \quad 20.00$

$2.50 \quad 22.50$

$3.00 \quad 27.50$

15.00

27.50

32.50

$1.50 \quad 12.50$

$1.75 \quad 15.00$

$2.25 \quad 20.00$

$3.00 \quad 27.50$

$3.75 \quad 35.00$

$4.75 \quad 45.00$

$6.75 \quad 65.00$

WEEPING TREES

MORUS tatarica pendula (Tea's Weeping Mulberry)

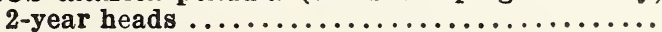

ULMUS campestris (Camperdown Elm)

2-year heads

2.25
100.00

125.00

175.00

250.00

325.00

425.00
12.50

17.50

20.00

25.00

15.00

20.00

25.00

25.00
25.00

30.00

20.00

25.00

\section{APPLES \\ Duchess \\ Fameuse \\ Grime's Golden \\ Jonathan \\ MeIntosh \\ Yellow Transparent}

\section{FRUITS (Bearing Size)}

\author{
Northern Spy Red June \\ N. W. Greening Maiden Blush \\ Talman Sweet Peerless \\ Wealthy \\ Winesap \\ Delicious
}


Each

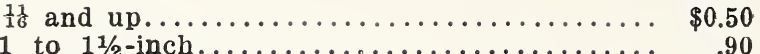

$11 / 2$ to 2 -inch..................... 1.35

APPLES (Dwarf)

Northern Spy

MeIntosh

Yellow Transparent

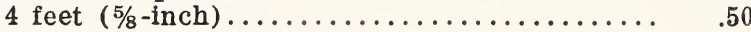

CRABS

Whitney

Hyslou

Red Siberian

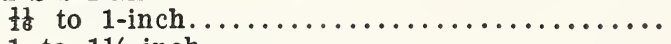

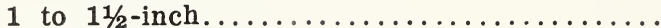

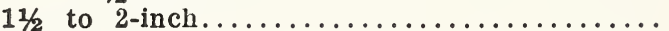

2 -inch

CHERRIES

Yellow Siberian

Early Strawberтy

.60

1.00

1.50

2.25
Per 10 Per 100

$\$ 4.50 \$ 40.00$

$8.50 \quad 80.00$

$12.50 \quad 115.00$

Early Richmond

Montmorency

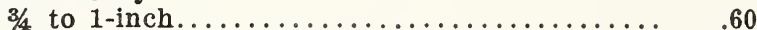

5.00

4.50

1.25

$11 / 2$-inch

2.25

2-inch

4.00

\section{PEARS}

$\begin{array}{ll}\text { Kiefier } & \begin{array}{l}\text { Flemish Beauty } \\ \text { Beckel } \\ \text { Duchett }\end{array} \\ \text { Sheldon }\end{array}$

$\frac{1}{16}$ to 1 -inch

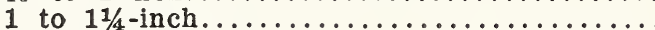

$11 / 2$ to 2 -inch

PEARS (Dwarf)

Flemish Beauty

Duchess

PLUMS

4 feet $(5 / 8-$ inch $)$

Bartlett

Green Gage

Burbank

Tonka

Abundance

$3 / 4$ to 1 -inch.

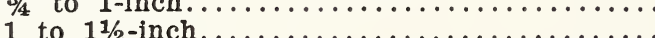

$11 / 2$ to 2 -inch

2.25

SMALL FRUITS
Shrop Damsen

Monitor

Opata

Hansa

\section{BLACKBERRIES}

\section{Snyder}

Eldorado

\section{Mersereau}

3-year

\section{CURRANTS}

\section{President Wilder}

Fay's Prolific

\section{Cherry}

2-year

\section{G00SERERRIES}

\section{Downing}

Champion

Josselyn

$$
\text { 2-year }
$$




$\begin{array}{lll}\text { Each } & \text { Per } 10 & \text { Per } 100 \\ \$ 0.20 & \$ 1.75 & \end{array}$

3-year Agawam

Moore's Early

Niagara

RASPBERRIES

Columbia

Cumberland

Golden Queen

2-year

STRA WBFRRIES

Standard varieties

Everbearing

\section{Worden}

$2.50 \$ 22.00$

.30

.12

1.00

8.00

.$\$ 1.00$ per $100 ; \$ 5.00$ per 1,000

3.00 per $100 ; 25.00$ per 1,000

\section{SHRUBS}

ACANTHOPANAX pentaphyllum (Aralia pentaphylla)

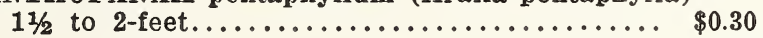

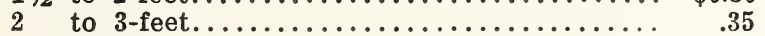

3 to 4 -feet..........................

AMELANCHIER canadensis (Juneberry)

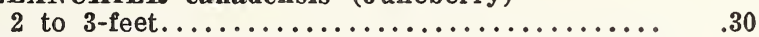

Per 10 Per 100

AMYGDALUS nana (Double White and Pink Almond)

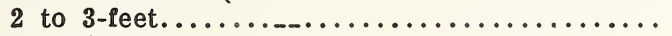

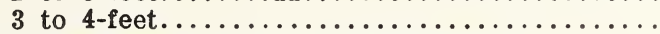

ARALIA spinosa (Devil's Walkingstick)

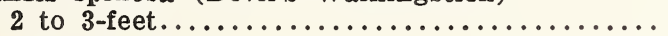

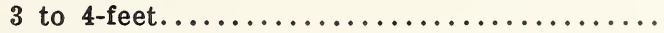

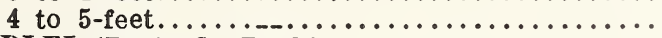

Each Per 10

$\$ 2.50$

3.00

$\$ 20.00$

3.50

25.00

30.00

2.75

25.00

4.00

35.00

$.60 \quad 5.00$

.50

4.00

.60

5.00

.75

6.00

BUDILEI (Butterfly Bush)

3-year

.35

3.00

25.00

BERBERIS thunbergi (Japanese Barberry)

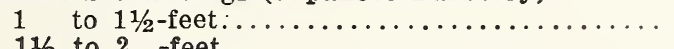

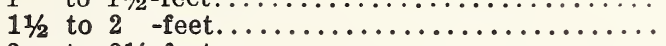

2 to $21 / 2$-feet.

$21 / 2$ to 3 -feet..................... $\quad .40$

1.50

1.75

2.50

3.50

Thunb. atropurpurea (purpleleaf Japanese Barberry)

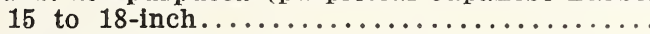

$11 / 2$ to 2 -feet..........................

CORNUS alba sibirica (Red Dogwood)

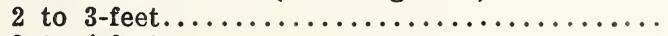

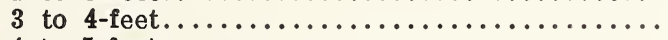

4 to 5 -feet.

.60

5.00

2.00

2.50

3.50

paniculata (Gray Dogwood)

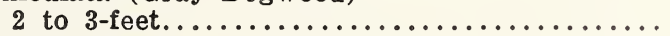

3 to 4-feet

stolonifera (Red osier Dogwood)

20.00

stolonifera flav. lutea (Goldentwig Dogwood)

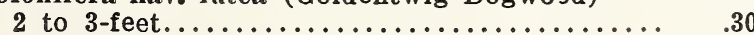

2.50

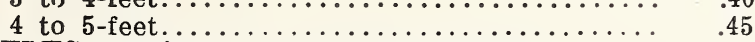

CORYLUS americana (American Hazelnut)

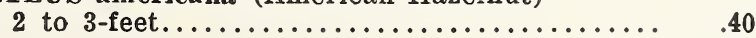

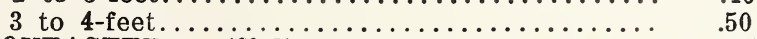

3.50

4.00

COTONEASTER acutifolia (Peking Cotoneaster)

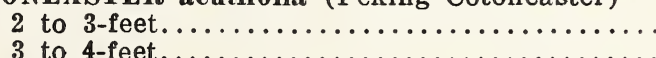

CRATEAGUS coceinea (Scarlet Fruited Hawthorn)

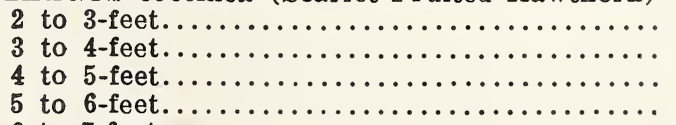

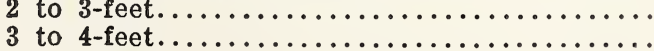


cras galli (Cockspur Thorn)

2 to 3 -feet.

3 to 4 -feet.

oxyacantha (English Hawthorn)

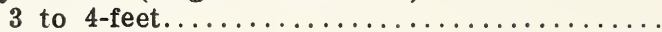

4 to 5 -feet

5 to 6 -feet.

oxyacantha pauli (Paul Scarlet Hawthorn)

5 to 6 -feet.

CYDONIA japonica (Flowering Quince)

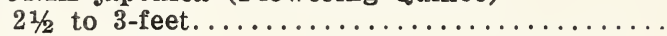

3 to 4 -feet.

DEUTZIA scabra crenata

3 to 4 -feet.

Pride of Rochester

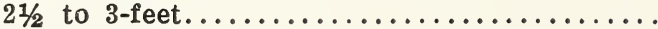

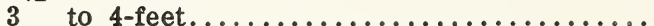

ELEAGNUS angustifolia (Russian Olive)

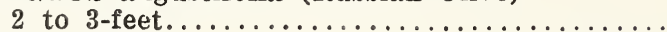

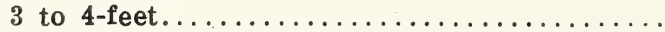

4 to 5 -feet

EUONYMUS alatus (Strawberry Bush)

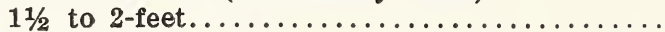

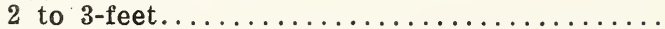

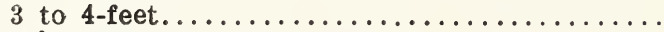

americana (Brook Euonymus)

europaeus (European Burningbush)

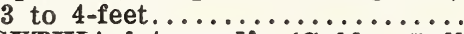

FORSYTHIA intermedia (Golden Bell)

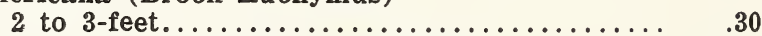

syriacus (tree form Double Assorted Althea)

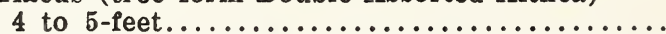

HYDRANGEA arborescens grandiflora (Snowball Hydrangea)

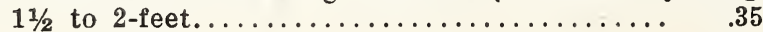

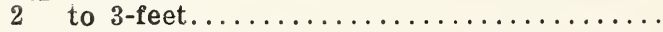

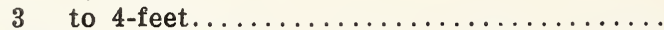

paniculata grandiflora (Bush Form) (Large

Flowering Hydrangea)

2 to 3 -feet.

3 to 4 -feet.

paniculata grandiflora (Tres Form)

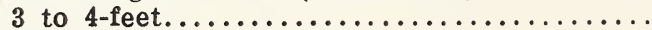

KOLKWITZIA (Beauty Bush)

$11 \%$ to 2 -feet.

LIGUSTRUM amurense (North Amur Privet) Each

$11 / 2$ to 2 -feet.................. $\$ 0.15$

2 to 3 -feet (Heavy)............... .20

3 to 4 -feet (Heavy) ................25
Each

$\$ 0.65$

.80

.60

.75

1.25

1.25

.35

.40

.30

.25

.30

.30

.35

.45

.50

.60

.75

.40

.25

.30

.35

.30

.25

.30

.35

.40

.50

.60

.35

.45

Per 10

$\$ 6.00$

7.50

5.50

6.50

10.00

3.00

3.50

2.50

22.00

2.25

2.50

20.00

22.00

2.50

20.00

3.00

4.00

25.00

35.00

4.00

5.00

6.50

2.50

3.50

2.25

2.50

3.00

20.00

22.00

25.00

2.50

20.00

2.25

20.00

2.50

22.00

3.00

25.00

3.50

4.00

5.00

3.00

25.00

4.00

30.00

.45

.40

.45

.85

1.00

Per 10

$\$ 1.00$

1.20

1.50
2.50

3.00

3.50

3.00

3.50

25.00

7.50

9.00

20.00

25.00

30.00 30.00

er 100

$\$ 8.00$

10.00

12.00
Per 1000

$\$ 60.00$

80.00 100.00 
ibota (Chinese Privet)

2 to $21 / 2$-feet. . . . . . . . . . . . . $\ldots \ldots \ldots \ldots$

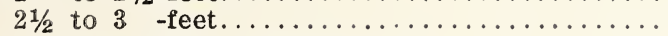

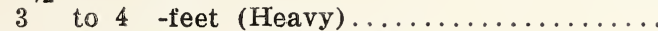

ibota regelianum (Regel's Privet)

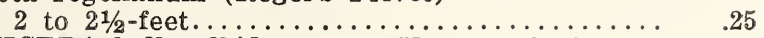

LONICERA bella albida (White Honeysuckle)

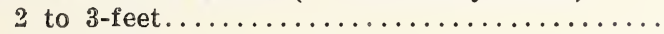

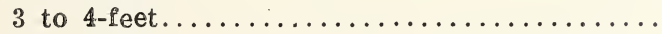

4 to 5 -feet.

5 to 6 -feet.

bella chrysantha

$2 \frac{1}{2}$ to 3 -feet

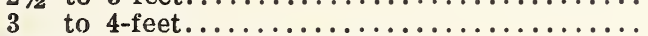

grandiflora (Pink Honeysuckle)

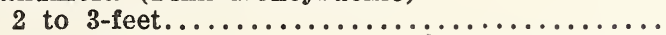

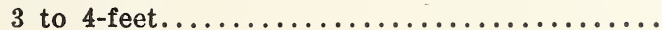

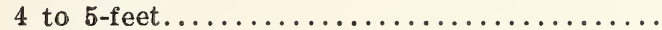

5 to 6 -feet.

morrowi

$21 / 2$ to 3 -feet.

3 to 4 -foet....

tatarica grandiflora rosea (Pink Tatarian Honeysuckle)

3 to 4-feet.

MAHONIA Aquifolium (Oregon Hollygrape)

1 to $11 / 2$-feet $B \& B . . . \ldots \ldots \ldots \ldots \ldots \ldots$

MALUS ioensis plena (Bechtel's Double Flowering Crab.)

2 to 3 -feet.

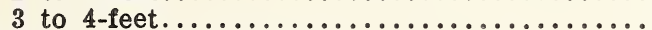

loensis (Wild Crab Apple)

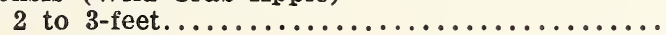

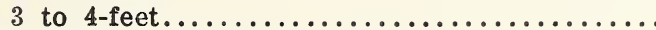

floribunda (Japanese Fl. Crab)

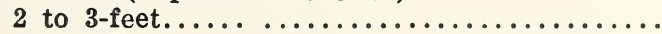

3 to 4 -feet.

floribunda atrosanguinea (Carmine Crab)

4 to 5 -feet. parkmani

2 to 3 -feet.

3 to 4 -feet.

4 to 5 -feet.

purpurea (Purple Leaf Crab)

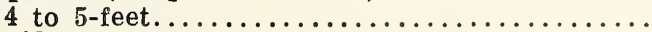
scheideckeri

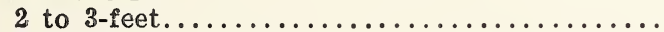

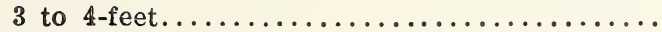

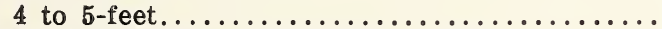

spectabilis (Chinese Flowering Crab)

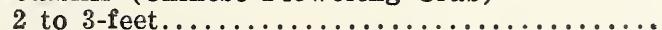

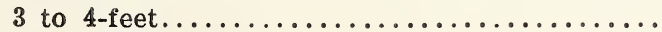

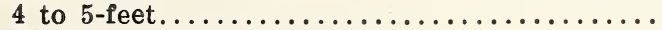

MORUS alba (Russian Mulberry)

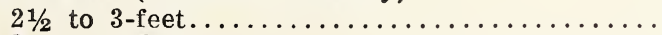

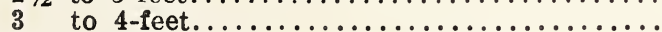

PHILADELPHUS avalanche (Dwarf Mockorange)

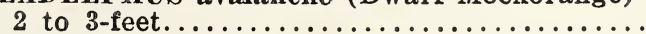

coronarius (Sweet Mockorange)

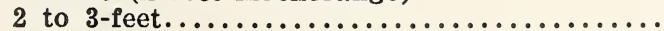

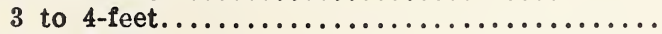

4 to 5 -feet.

cor. Glacier

2 to 3 -feet.
Each

.25

.20

.25

.30

.40

.25

.30

.25

.30

.35

.50

.30

.35

.35

1.25

.60

.10

.50

.60

.75

.75

.75

1.00

1.50

.75

.60

.75

1.00

.60

.75

1.00

.15

.20

.30

.25

.30

.40

.35
Per 10

$\$ 1.20$

1.50

2.00

2.25

1.50

2.00

2.50

3.50

2.00

2.50

2.00

2.50

3.00

4.00

2.50

3.00

3.00

25.00

10.00

4.00

5.00

3.50

4.00

5.00

6.00

6.00

7.50

5.00

6.00

5.00

6.00

1.25

1.50

8.00

12.00

2.50

2.25

2.50

3.50

20.00

22.00

30.00

3.00 
grandiflorus (Large Fl. Mockorange)

Each

2 to 3 -feet......................... $\$ 0.25$

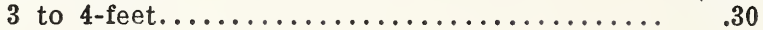

4 to 5 -feet...........................

\section{lemoinei}

2 to 3 -feet

nivalis

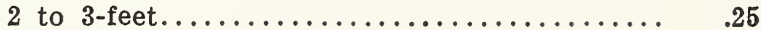

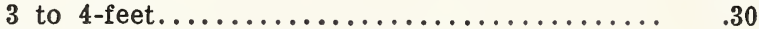

virginal (Double Fl. Sweet Scented)

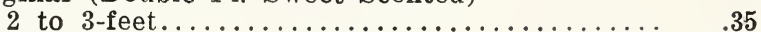

3 to 4 -feet..........................

PHYSOCARPOS opulifolius (Ninebark)

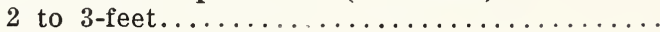

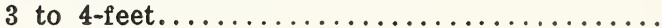

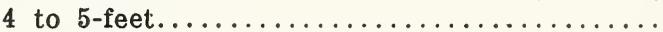

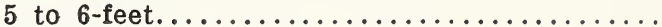

opulifolius nana

$11 / 2$ to 2 -feet

POTENTILLA fruticosa (Shrubby Cinquefoil)

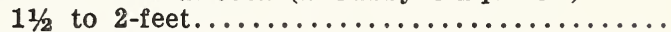

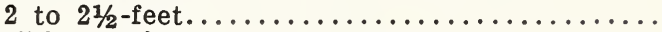

PRUNUS americana (Native Plum)

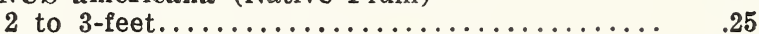

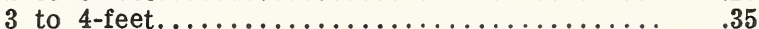

4 to 5 -feet........................... $\quad .40$

othello (Purple Leaved Plum)

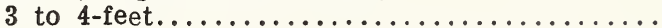

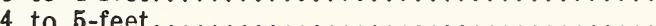

virginiana

4 to 5 -feet....................... 50

serotina

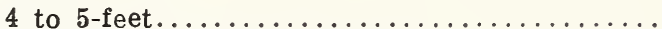

triloba plena (Double Fi. Plum)

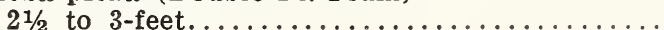

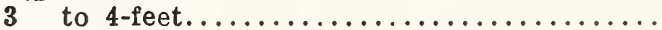

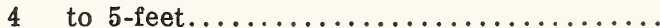

RHAMNUS frangula (Glossy Buckthorn)

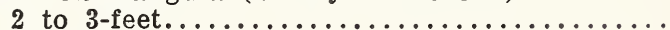

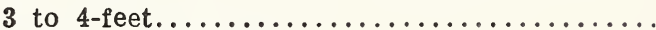

Cathartica (Buckthorn)

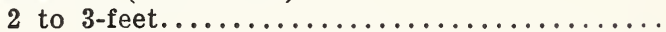

3 to 4 -feet.

RHODOTYPOS kerriodes

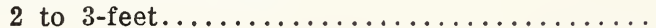

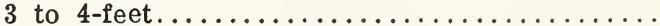

RHUS aromatica (fragrant sumac)

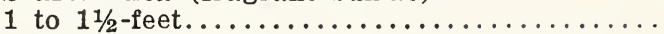

copallina (shining sumac)

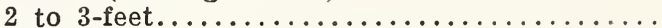

cotonoides (American Smoke tree)

2 to 3 -feet........................ 50

glabra (Smooth Sumac)

3 to 4 -feet....................... $\quad .30$

typhina (Staghorn Sumac)

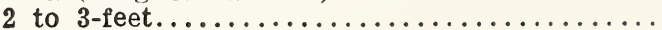

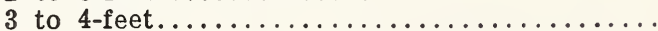

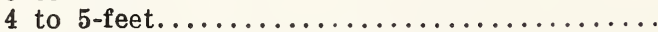

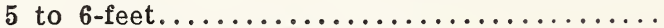

typhina laciniata (Cut-leaved Sumac)

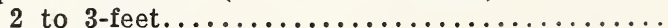

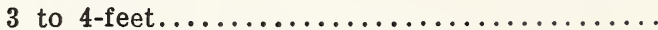

RIBES Alpinum (Mountain Currant)

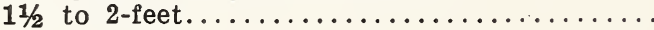

2 to $21 / 2$-feet.
Per 10

$\$ 2.25$

2.50

3.50

2.50

2.25

2.50

3.00

4.00

2.00

2.50

3.00

3.50

3.00

3.00

3.50

2.00

3.00

3.50

15.00

25.00

30.00

4.50

5.50

4.00

4.00

3.50

4.00

5.00

1.75

2.00

15.00

17.50

1.75

2.00

15.00

17.50

3.00

25.00

4.00

35.00

3.00

25.00

3.00

4.00

2.50

22.00

2.00

2.50

3.00

3.50

.35

.45

16.00

20.00

25.00

35.00 
2 to 3 -feet....................... $\$ 0.30$

3 to 4 -feet............................ $\quad .40$

A blanda (Meadow Rose)

carolina (Swamp Rose)

$3.00 \quad 25.00$

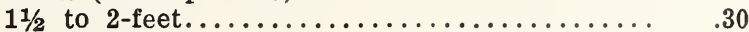

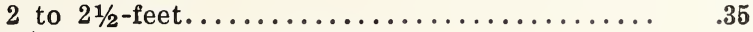

Grootendorst (Bright Crimson)

hansa (red)

$11 / 2$ to 2 -feet.......................... 40

hugonis (yellow)

multiflora japonica (Jap Rose)

rubiginosa (Sweetbrier)

2 to 3 -feet..........................

rubrifolia (Redleaf Rose)

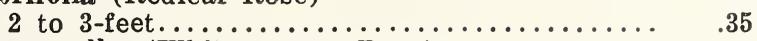

rugosa alba (White rugosa Rose)

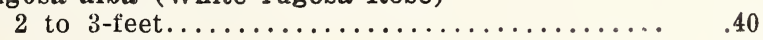

3.50

3 to 4 -feet.................

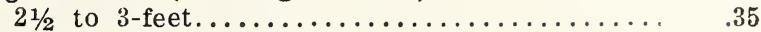

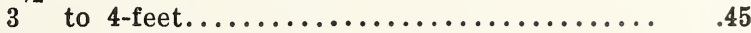

Sir Thomas Lipton, double, snow-white

Conrad F. Meyer, silvery-pink

2-year
Setigera (Prairie Rose)

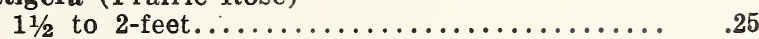

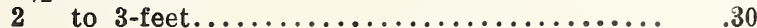

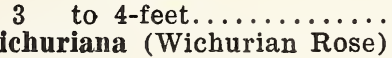

ROSES, Hybrid Tea (Field Grown)

Columbia (pink)

Duchess of Wellington (yellow)

Etoile de France (red)

Gruss an Teplitz (crimson)

Kaiserin Auguste Viktoria (white)

Lady Ashtown (pink)

Lady Hillingdon (Apricot yellow)

Los Angeles (golden pink)

Louise Catherine Breslau (coral red)

Mme. Caroline 'Testout (pink)

Mme. Edouard Herriot (pink)

Padre (red)

Pink Killarney (pink)

Pink Radiance (pink)

Red Radiance (red)

Souvenir de Claudius Pernet (yellow)

2-year

ROSES, Hybrid Perpetual (Field Grown)

Frau Karl Druschki (white)

Gen. Jacqueminot (bright crimson)

Harrison's Yellow (deep golden yellow)

Luxembourg (yellow)

Magna Charta (pink, large flowering)

Mme. Plantier (white)

Mrs. Joln Laing (soft pink) 
Paul Neyron (pink)

Persian Yellow (yellow)

Vlrich Brunner (red)

2-year

ROSES, Climbing

American Beauty (red)

Crimson Rambler (deep crimson)

Dorothy Perkins (pink and white)

Dr. Van Fleet (shell pink)

Excelsa (red)

Flower of Fairfield (crimson, everblooming)

Gardenia (yellow)

Hiawatha (crimson, white eye)

Mary Wallace (rose pink)

Paul's Scarlet Runner (scarlet)

Prairie Queen (pink)

Poserie (red)

Seven Sisters (pink)

Silver Moon (white)

Source Dor (yellow)

Tousandschon (pink and white)

2-year .............................40

SAMBUCUS acutiloba (Cutleaf Elder)

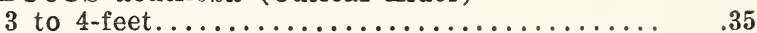

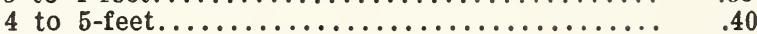

Crmadensis (American Elder)

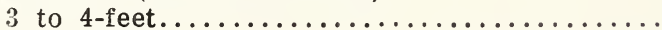

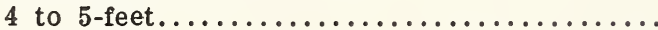

5 to 6 -feet.

canadensis aurea (Golden Elder)

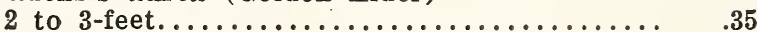

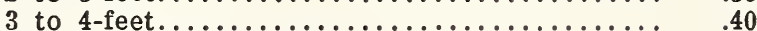

pubens (Red berried Elder)

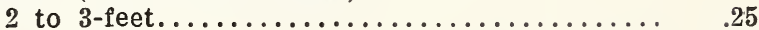

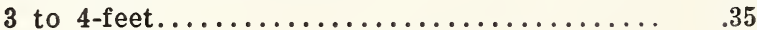

SORBARIA sorbifolia (Ural False Spirea)

2 to 3 -feet......................... 30

SPIRAEA Anthony Waterer

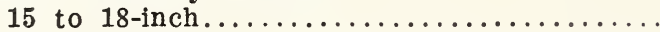

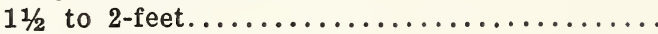

arguta (Garland Spirea)

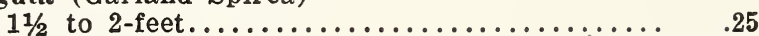

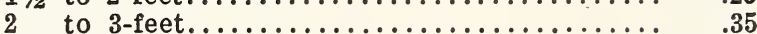

Bethlehemensis (pink)

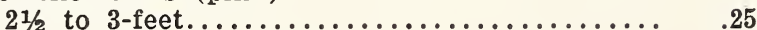

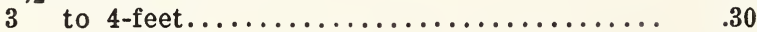

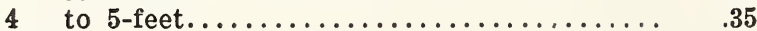

Billardi

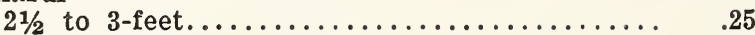

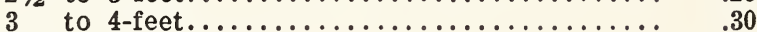

froebeli (Dwarf Pink)

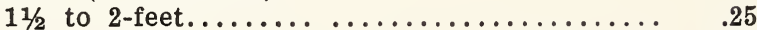

2 to $21 / 2$-feet.................................

opulifolia (see Physocarpos)

Thunbergi

2 to 3 -feet................................

trichocarpa (Korean Spirea)

2 to 3 -feet...........................

van houttei (Bridal Wreath)

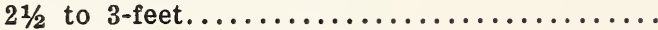

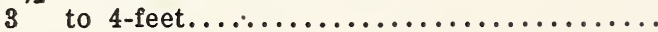

4 to 5 -feet (Heavy) $\ldots \ldots \ldots \ldots \ldots \ldots \ldots \ldots \ldots$ 
SYMPHORICARPOS racemosus (Snowberry)

$\begin{array}{rrr}\text { Each } & \text { Per 10 } & \text { Per } 100 \\ \$ 0.20 & \$ 1.50 & \$ 12.00 \\ .25 & 2.00 & 16.00 \\ .35 & 3.00 & 25.00 \\ & & \\ .20 & 1.50 & 12.00 \\ .25 & 2.00 & 15.00 \\ .50 & 4.00 & 35.00 \\ .55 & 4.50 & \\ & & \\ .35 & 3.00 & 25.00 \\ .40 & 3.50 & 30.00\end{array}$

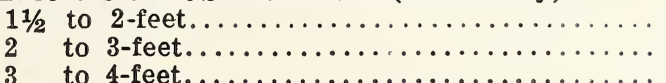

vulgaris coralberry (Indian Currant)

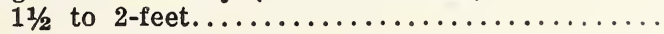

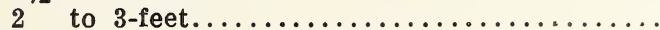

SYRINGA vulgaris alba (White Lilac)

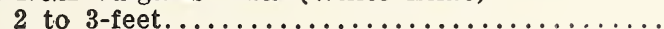

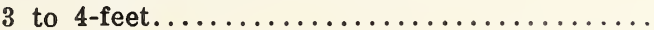

vulgaris (Common Purple Lilac)

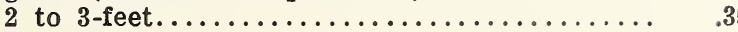

3 to 4 -feet......................... $\quad .40$

30.00

vulgaris (Named varieties)

Mme. C. Perier (double white)

Mme. Lemoine (white)

Marie Legraye (white)

Jean Mace (double, bluish)

Lamartine (single, rosy mauve)

Clande Bernard (bright lilac)

Chas. Joly (deep violet)

Michael Buchner (lilac purple)

Vauban (pinkish mauve)

Ludwig Spaeth (single, purple)

Chas. X (reddish purple)

Jacques Calot (large rosy-pink)

Vestal (large white)

Belle de Nancy (satiny rose)

Hugo Koster (purplish lilac)

1 to $11 / 2$-feet......................... $\quad .40$

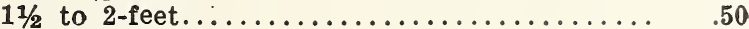

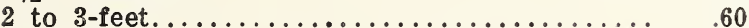

3 to 4 -feet.......................... $\quad .75$

Japonica

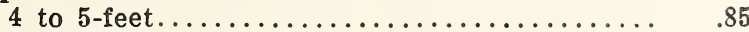

3.50

30.00

$4.00 \quad 35.00$

$5.50 \quad 50.00$

$6.50 \quad 60.00$

chinensis rothomagensis (Rouen Lilac)

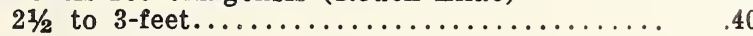

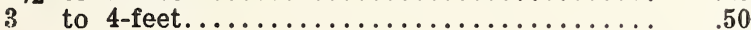

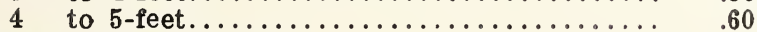

7.50

3.50

4.00

5.00

30.00

35.00

45.00

Persica (Persian Lilac)

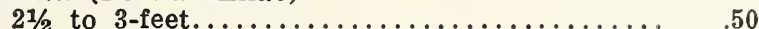

4.00

35.00

3 to 4 -feet............................

4.50

40.00

villosa (Late Lilac)

3 to 4 -feet............................ .45

4.00

TAMARIX Amurense (Amur River Tamarisk)

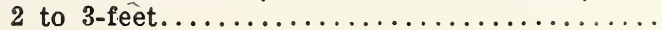

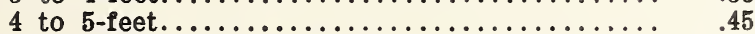

hispida aestivalis (Kashgar Tamarisk)

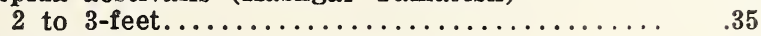

3 to 4 -feet.........................45

3.00

35.00

VIBURNUM dentatum (Arrow-wood)

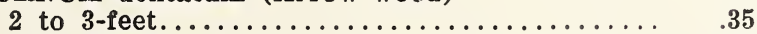

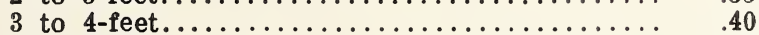

3.50

25.00

30.00

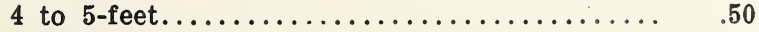

3.00

25.00

$3.50 \quad 30.00$

$4.00 \quad 35.00$

acerifolinm (Mapleleaf Spirea)

$11 / 2$ to 2 -feet........................40

Americanum (American H. B. Cranberry)

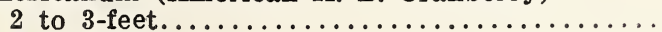

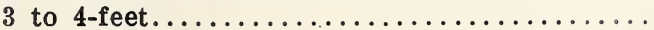

4 to 5-feet. 
2 to 3 -feet...................... $\$ 0.45$

3 to 4 -feet........................ $\quad .50$

lentago (Nannyberry)

2 to 3 -feet......................... $\quad .40$

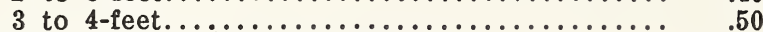

$\$ 4.00 \$ 35.00$

4.50

40.00

opulus (European H. B. Cranberry)

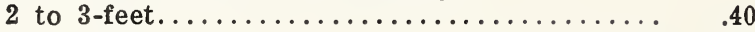

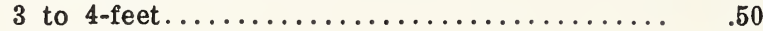

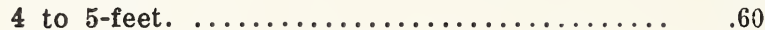

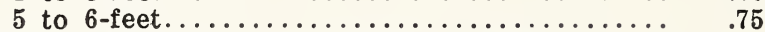

3.50

4.50

opulus nanum (Dwarf Cranberry Bush)

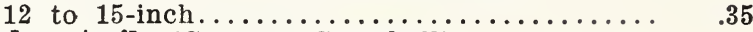

opulus sterile (Common Snowball)

2 to 3 -feet.

3 to 4 -feet.

pubescens. This handsome shrub, though not well known, should be more extensively used. Its good foliage and glossy black berries make it exceedingly attractive. It is on $\theta$ of the best of this exceedingly attractive group of plants.

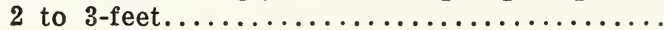

WEIGELA Abel Carriere (Rosy Carmine)

Eva Rathke (red)

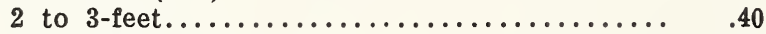

3 to 4 feet.

rosea florida (pink)

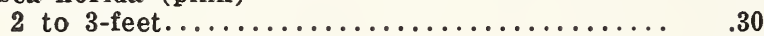

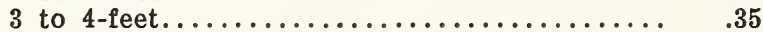

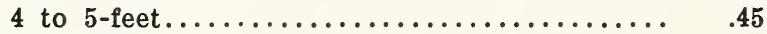

4.50

2.50

20.00

\section{CLIMBING VINES}

AMPELOPSIS quinquefolia engelmanni (Englemann's Ivy)

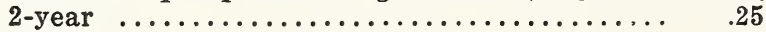

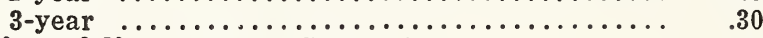

quinquefolia (Virginia Creeper)

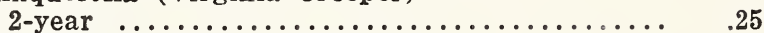

$2.00 \quad 15.00$

$2.50 \quad 20.00$

tricuspidata veitchi (Boston Ivy)

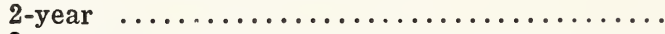

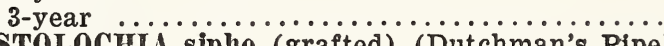

ARISTOLOCHIA sipho (grafted) (Dutchman's Pipe)

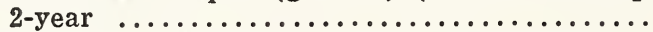

CELASTRUS orbiculatus (Oriental Bittersweet)

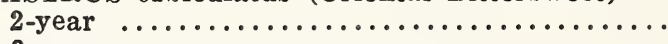

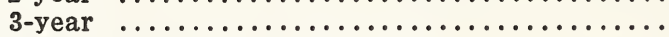

Scandens (American Bittersweet)

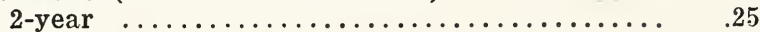

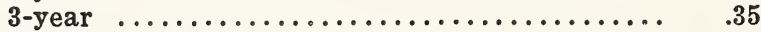

$2.00 \quad 15.00$

CLEMATIS Jackmanni (Purple, Large Flowering)

2-year

Mme. Andre

2-year .............................6

paniculata (Sweet Autumn Clematis)

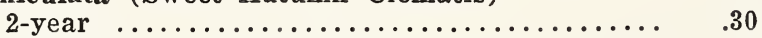

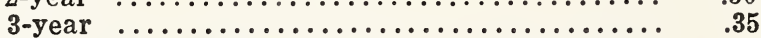

LONICERA Jap. halleana (Hall Jap. Honeysuckle)

2-year ................................. 
WISTERIA sinensis grafts (Chinese Blue Wisteria)

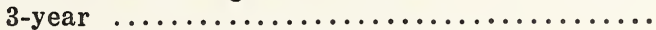

sinensis alba (White Chinese Wisteria).........

\section{RHUBARB}

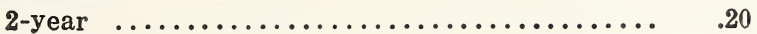

\section{ASPARAGUS}

Conovers, Palmetto and Washington

2-year

\section{EVERGREEN}

\section{Prices include B. \& B.}

ABIES Concolor (White Fir)

$11 / 2$ to 2 -feet............

Balsamea (Balsam Fir)

$21 / 2$ to 3 -feet...................... 3.00

BUXUS sempervirens (Dwarf Boxwood)

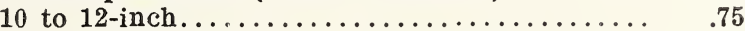

18 to 24 -inch B\&B......................... 2.00

JUNIPERUS communis (Var. Canadensis)

(Prostrate Juniper)

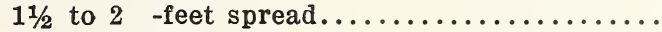

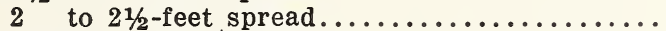

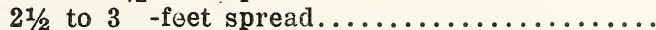

Each

$\$ 3.00$

Per 10

$\$ 27.50$

Per 100

Per 100 Per 1000

$\$ 2.00 \quad \$ 12.00$

Chinensis Pfitzeriana (Pfitzer Juniper)

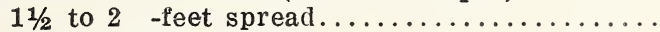

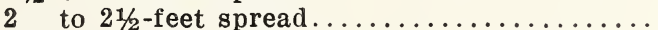

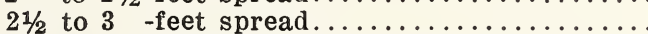

Sabina tamariscifolia (Tamarisk Savin)

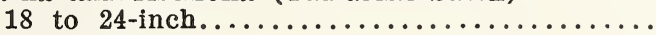

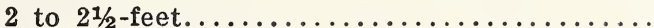

Virginiana (Red Cedar)

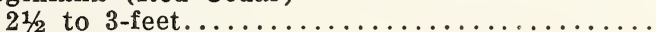

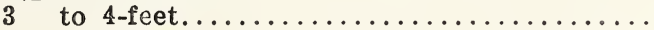

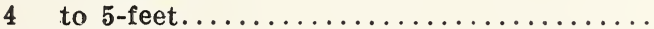

5 to 6 -feet.

Virginiana Cannarti

2 to $21 / 2$-feet...................... 3.25

$21 / 2$ to 3 -feet..................... 4.00

1.75

2.25

2.75

2.25

2.75

3.25

2.75

3.75

2.50

3.50

4.50

6.50

Virginiana glanea (Silver Red Cedar)

2 to 3 -feet.

4.00

3 to 4 -feet.

6.00

PICEA canadensis alba (White Spruce)

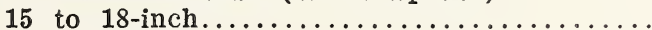

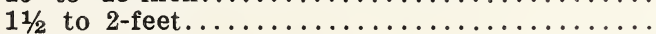

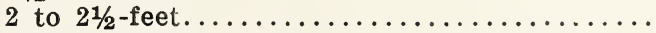

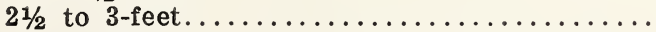

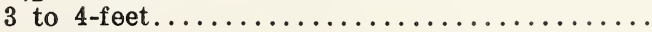

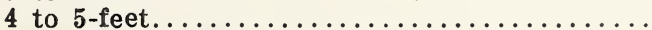

Canadensis Albertiana (Black Hills Spruce)

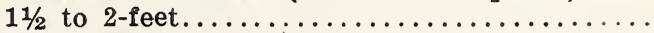

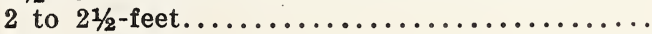

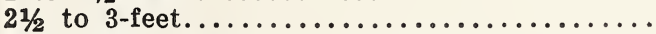

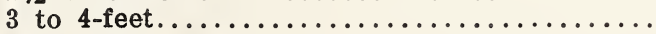

4 to 5 -feet. 
Excelsa (Norway Spruce)

$11 / 2$ to 2 -feet.

2 to $21 / 2$-feet

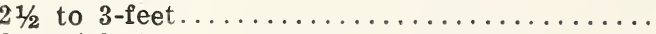

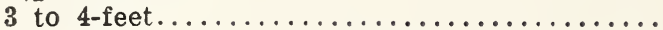

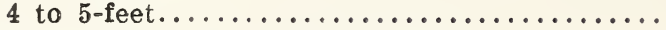

Pungens (Colorado Green Spruce)

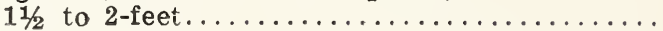

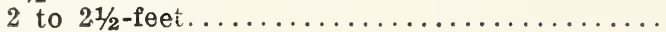

$21 / 2$ to 3 -feet

Pungens glauca (Colorado Blue Spruce)

1 to $1 \frac{1}{2}$-feet.

$11 / 2$ to 2 -feet.

2 to $21 / 2$-feet.

$21 / 2$ to 3 -feet.

PINUS Riontana Mughus (Mugho Pine)

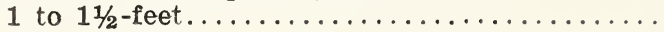

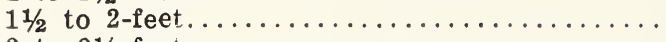

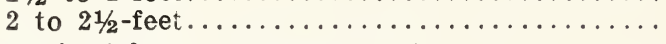

nigra Austriaca (Austrian Pine)

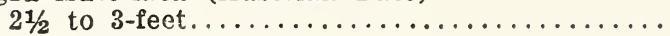

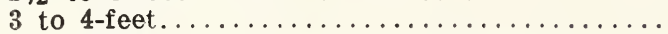

4 to 5 -feet.

Ponderosa (Western Pine)

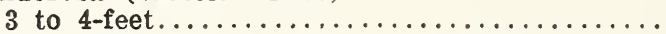

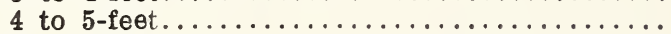

strobus

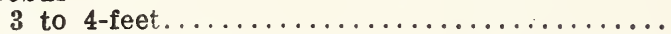

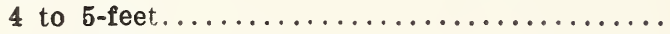

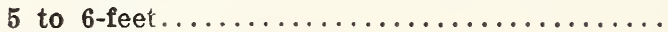

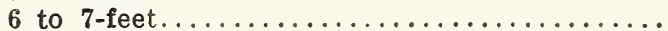

sylvestris (Scotch Pine)

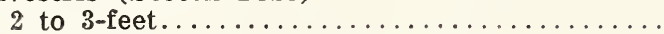

3 to 4 -feet.

Tanyosho (Japanese Table Pine)

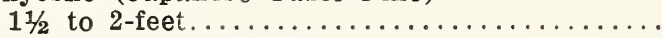

PSEUDOTSUGA Canadensis (Douglas Fir)

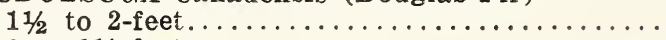

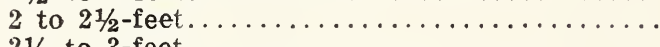

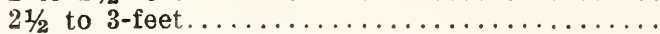

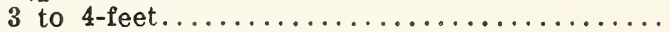

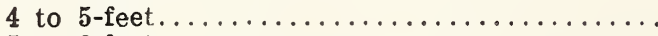

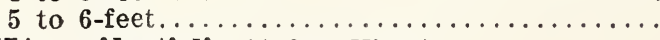

THUJA occidentialis (Arbor Vitae)

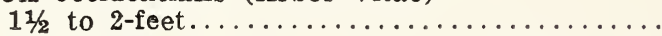

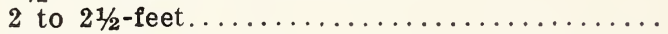

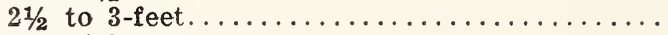

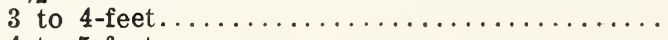

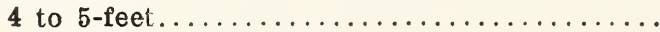

occ. globosa.

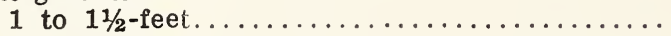

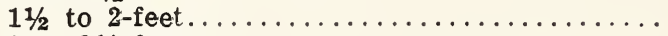

2 to $21 / 2$-feet

occ. pyramidalis

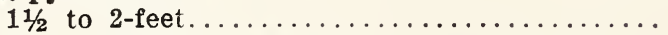

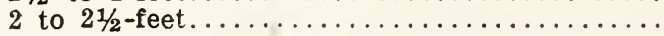

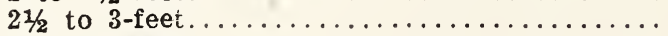

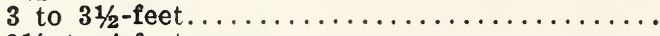

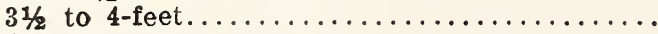

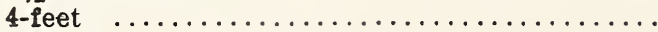

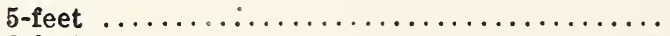

6-leet
Each

$\$ 0.90$

1.50

1.75

2.50

3.25

1.75

2.25

3.25

3.75

4.25

6.50

7.50

$2.00 \quad 17.50$

2.25

4.25

3.75

4.25

6.75

3.50

5.00

3.25

4.75

5.75

6.75

$2.00 \quad 17.50$

$2.75 \quad 25.00$

$4.25 \quad 40.00$

$1.75 \quad 15.00$

$2.50 \quad 20.00$

$3.50 \quad 30.00$

$4.50 \quad 40.00$

$\mathbf{5 . 5 0} \mathbf{5 0 . 0 0}$

6.50

$1.25 \quad 10.00$

$1.50 \quad 12.50$

$1.75 \quad 15.00$

$2.50 \quad 22.50$

$3.50 \quad 30.00$

$1.25 \quad 10.00$

$1.75 \quad 15.00$

2.2520 .00

$1.50 \quad 12.50$

$1.75 \quad 15.00$

$2.25 \quad 20.00$

$3.25 \quad 30.00$

$3.75 \quad 35.00$

$5.25 \quad 50.00$

7.50

9.00 
AGROSTEMMA (Rose Campion).

Each

$\$ 0.15$

ACHILlEA Ptarmica (The Pearl)

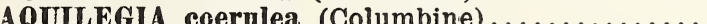

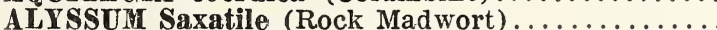

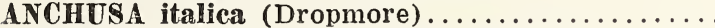

ANTHEMIS Kelwayi (Golden Marguerite).........

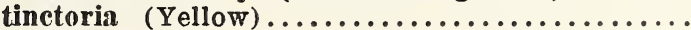

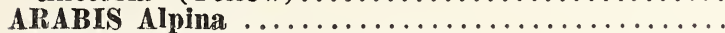

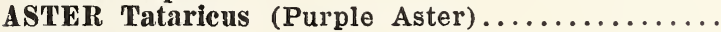

ASTILBE

AUBRIETIA (Large Flowering Hybrids)

BELLIS Perennis (English Daisy) .............

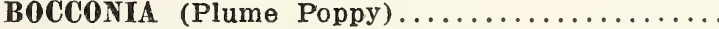

BOLTONIA asteroides (White) (False Chamomile). latisquama (pink)

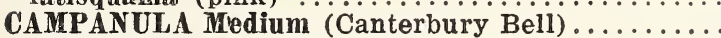

CARNATION

CENTAUREA Montana (Blue Corn Flower).......

CERASTIUM Tomentosum (Snow in Summer)....

CHEIRANTHUS allionii $\ldots \ldots \ldots \ldots \ldots \ldots \ldots$

CHRYSANTHEMUM Maximum (Shasta Daisy)....

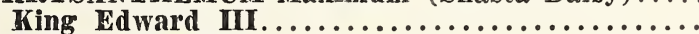

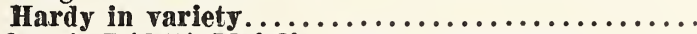

CONVALLARIA Majalis (Lily of the Valley).......

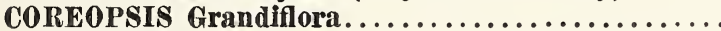

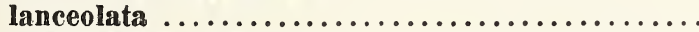

DELPHINIUM Belladonna (Larkspur) Light Blue. .

formosum

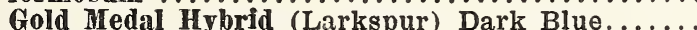

DIANTHUS Alwoodi . . . . . . . . . . . . . . .

atro-sanguinia

Barbatus

plumarius (Hardy pinks)

DICENTRA spectabilis (Bleeding Heart)

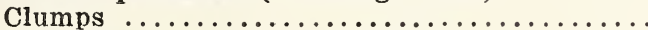

DIGITALIS monstrosa (Fox Glove) .............

ECHIN0PS Ritro (Globe Thistle) ..............

FUNKIA subcordata (Day Lily) ..............

GAILLARDIA grandiflora (Blanket Flower)......

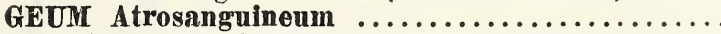

IELIANTHUS giganteus (Wild Sunfiower).......

HEMEROCALLIS (Lemon Lily) ...............

HESPERIS matronalis (Purple Spike Flowers)....

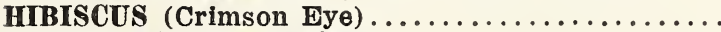

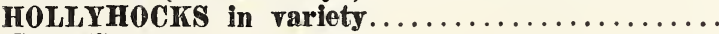

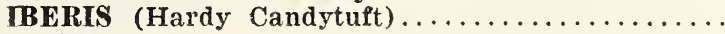

IRIS Gierman

Ambassadeur (S. lavender F. deep violet) ........

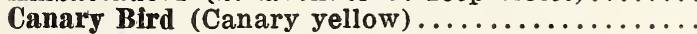

Chas, Dickens (S. and F. light lavender)........

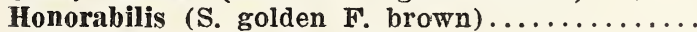

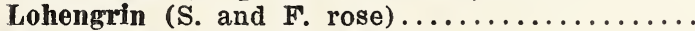

Mme. Chereau (S. white F. frilled blue) ..........

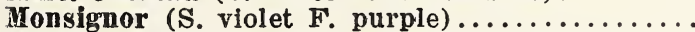

Mother of Pearl (light lavender displaying iri-

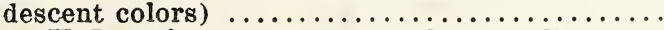

Mrs. H. Darwin (S. white $F$. violet tinted) ......

Pallida Dalmatica (S. pale lavender F. lavender)

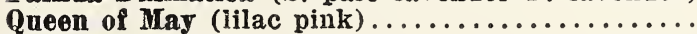

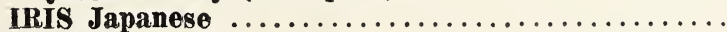

LATHYRUS Sweet Pea.
Per 10 Per 100

$\$ 1.25 \$ 10.00$

$1.25 \quad 10.00$

$1.25 \quad 10.00$

$1.25 \quad 10.00$

$1.25 \quad 10.00$

$1.25 \quad 10.00$

$1.25 \quad 10.00$

$1.25 \quad 10.00$

$\begin{array}{ll}1.25 & 10.00\end{array}$

$4.00 \quad 35.00$

$\begin{array}{ll}1.25 & 10.00\end{array}$

$1.25 \quad 10.00$

$1.50 \quad 12.00$

$1.50 \quad 12.00$

$\begin{array}{ll}1.25 & 10.00\end{array}$

$1.50 \quad 12.00$

$1.25 \quad 10.00$

$1.25 \quad 10.00$

$\begin{array}{ll}1.25 & 10.00\end{array}$

$1.25 \quad 10.00$

$1.25 \quad 10.00$

$\begin{array}{ll}1.25 & 10.00\end{array}$

$1.75 \quad 15.00$

$1.25 \quad 10.00$

$1.25 \quad 10.00$

$1.25 \quad 10.00$

$1.50 \quad 12.00$

$1.25 \quad 10.00$

$\begin{array}{ll}1.50 & 12.00\end{array}$

$1.25 \quad 10.00$

$1.25 \quad 10.00$

$1.25 \quad 10.00$

$1.25 \quad 10.00$

$3.50 \quad 30.00$

$1.50 \quad 12.00$

$1.25 \quad 10.00$

$\begin{array}{ll}1.50 & 12.00\end{array}$

$1.25 \quad 10.00$

$1.50 \quad 12.00$

$1.25 \quad 10.00$

$1.75 \quad 15.00$

$\begin{array}{ll}1.25 & 10.00\end{array}$

$1.25 \quad 10.00$

$1.25 \quad 10.00$

$1.75 \quad 15.00$

$1.50 \quad 12.00$

$1.50 \quad 12.00$

$\begin{array}{ll}1.50 & 12.00\end{array}$

$1.50 \quad 12.00$

$1.50 \quad 12.00$

$1.50 \quad 12.00$

6.00

4.00

$1.50 \quad 12.00$

$1.50 \quad 12.00$

$1.50 \quad 12.00$

$2.00 \quad 15.00$

$1.25 \quad 10.00$ 


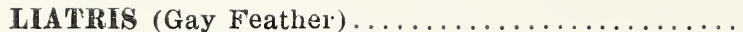

LILIUM regale (Regal Lily) ................

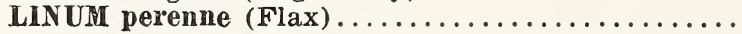

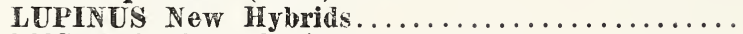

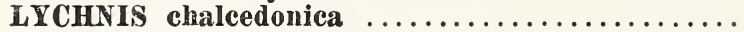

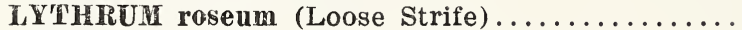

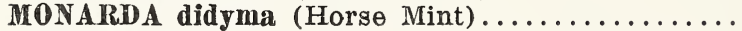

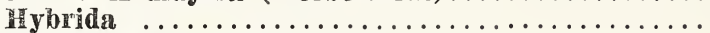

MYOSOTIS (Forget-me-not) $\ldots \ldots \ldots \ldots \ldots \ldots \ldots \ldots$

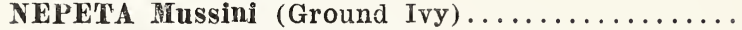

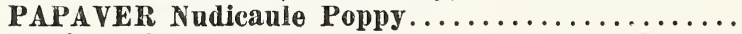
orientalis

PEONIES (in variety)

Louis Van Houtte (dark crimson)

Caroline Mather (red)

Couronne d'0r (white, red center)

Festiva Maxima (large white)

Edulis superba (soft pink, early)

Fragrans (red)

Duchess de Nemours (sulphur white, late)

Duke of Wellington (sulphur white, late)

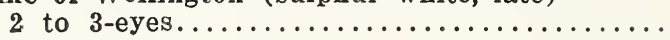

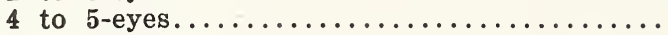

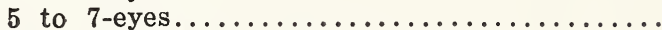

Baroness Schroeder (white, soft pink center)

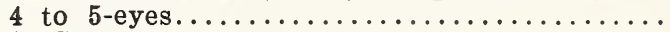

Felix Crousse (brilliant red)

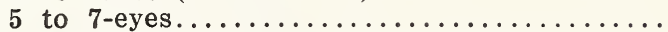

Karl Rosenfield (deep brilliant crimson)

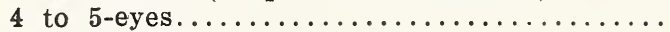

Mons. Jules Elie (pale lilac rose)

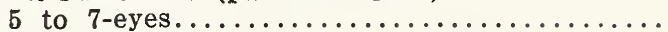

PHYSALIS Francheti (Chinese Lantern plant).... .

PHYSOSTEGIA Virginica (False Dragonhead).....

PLATYCODON grandiflora

(Chinese bell flower) $\ldots \ldots \ldots \ldots \ldots \ldots \ldots \ldots \ldots$

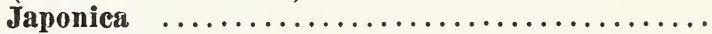

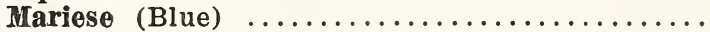

PHLOX in variety.

Beacon (cherry red)

Mrs. Charles Dorr (lavender)

Bridesmaid (white with crimson center)

Commander (crimson)

Champs Elysee (purplish)

Eclaireur (bright rose)

R. P. Struther (cherry red)

Mrs. Jenkins (large white, early bloomer)

Thor (salmon pink, red eye)

La Vague (lavender pink)

Baron von Dedem (brilliant red, salmon shadings)

Pantheon (rose pink)

Rynstrom (rose pink)

Prof. Virchow (sunset, Champs Elysee)

Elizabeth Campbell (pink)

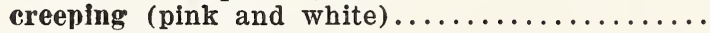

POLEMONIUM coeruleum . . . . . . . . . . . .

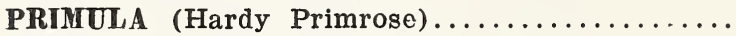

PYRETHRUM Hybridum (Painted Daisy)

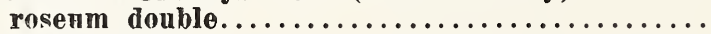

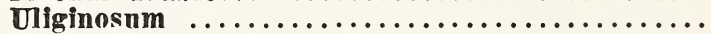

RANUNCULUS repens (Double Flowering Dwarf

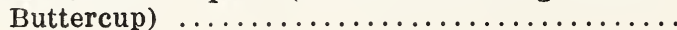

Each

$\$ 0.15$

.50

.15

.15

.15

.15

.15

.15

.15

.15

.15

.15

Per 10

$\$ 1.25$

4.00

1.25

1.25

1.25

1.25

1.25

1.25

1.25

1.25

1.25

1.25

Per 100

$\$ 10.00$

10.00

10.00

10.00

10.00

10.00

10.00

10.00

10.00

10.00

10.00

.35

.50

.60

3.00

4.00

5.00

25.00

35.00

$\mathbf{4 5 . 0 0}$

$1.25 \quad 10.00$

.75

6.00

55.00

1.00

9.00

.75

.15

6.00

1.25

1.25

1.25

1.25

1.25

2.00

55.00

10.00

10.00

.15

.15

.15

.25

10.00

10.00

10.00

15.00
.15

.15

.15

.15

.15

.15
1.25

1.25

1.25

10.00

10.00

10.00

1.25

1.25

10.00

10.00

1.25

10.00 


\begin{tabular}{|c|c|c|c|}
\hline RUDBECKIA purpurea (Purple Coneflower) ....... & $\underset{\$ 0.15}{\text { Each }}$ & $\begin{array}{l}\text { Per } 10 \\
\$ 1.25\end{array}$ & $\begin{array}{l}\text { Per } 100 \\
\$ 10.00\end{array}$ \\
\hline 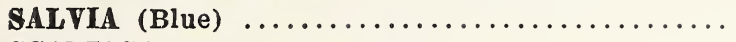 & .15 & 1.25 & 10.00 \\
\hline 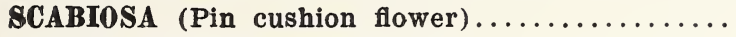 & .20 & 1.50 & 12.00 \\
\hline BEDUM spectabilis (Stone crop)............. & .15 & 1.25 & 10.00 \\
\hline $\begin{array}{l}\text { TRITOMA } \\
\text { TROLLIUS Led Hot Poker) } \ldots \ldots \ldots \ldots \ldots \ldots \ldots \ldots \ldots \ldots \ldots \ldots \ldots \ldots\end{array}$ & $\begin{array}{l}.25 \\
.20\end{array}$ & $\begin{array}{l}2.00 \\
1.75\end{array}$ & 15.00 \\
\hline 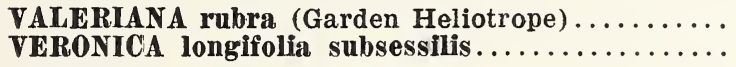 & $\begin{array}{l}.15 \\
.20\end{array}$ & $\begin{array}{l}1.25 \\
1.75\end{array}$ & $\begin{array}{l}10.00 \\
15.00\end{array}$ \\
\hline VERONICA spicata (Speedwell) ............. & .20 & 1.50 & 12.00 \\
\hline $\begin{array}{l}\text { VIOLA (Tufted Pansies) } \ldots \ldots \ldots \ldots \ldots \ldots \ldots \ldots \ldots \\
\text { YUCCA (Adam's Needle) }\end{array}$ & .15 & 1.25 & 10.00 \\
\hline 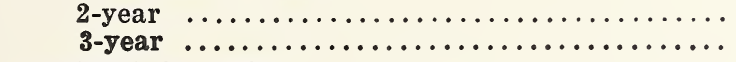 & .20 & $\begin{array}{l}1.50 \\
1.75\end{array}$ & $\begin{array}{l}12.00 \\
15.00\end{array}$ \\
\hline RASSES in variety. & .15 & 1.20 & \\
\hline
\end{tabular}

\section{BULBS}

TULIPS— $\$ 5.00$ per $100 ; \$ 40.00$ per 1,000 .

\section{FERTILIZER}

\section{VIGORO}

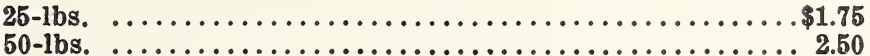

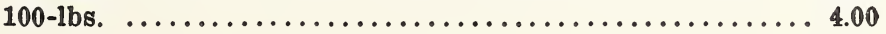

\section{BOXING and PACKING CHARGES}

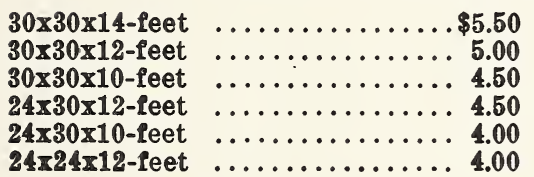

$30 \times 30 \times 14-$ feet

$30 \times 30 \times 12$-feet

$30 \times 30 \times 10$-feet

$24 \times 30 \times 12$-feet

24x24x12-peet

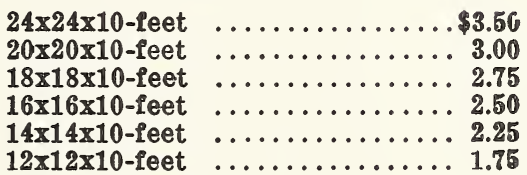

$24 \times 24 \times 10$-feet $\ldots \ldots \ldots \ldots \ldots \ldots 3.50$

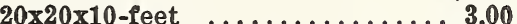

$18 \times 18 \times 10$-feet $\ldots \ldots \ldots \ldots \ldots \ldots 2.75$

$16 \times 16 \times 10$-feet

$12 \times 12 \times 10$-feet ............. 1.75 


\section{MEMORANDUM}



